\title{
Label-free detection of interleukin-6 using electrolyte gated organic field effect transistors
}

Chiara Diacci, Marcello Berto, Michele Di Lauro, Elena Bianchini, Marcello Pinti, Daniel Simon, Fabio Biscarini and Carlo A. Bortolotti

The self-archived postprint version of this journal article is available at Linköping University Institutional Repository (DiVA):

http:/ / urn.kb.se/ resolve?urn=urn:nbn:se:liu:diva- 141922

N.B.: When citing this work, cite the original publication.

Diacci, C., Berto, M., Di Lauro, M., Bianchini, E., Pinti, M., Simon, D., Biscarini, F., Bortolotti, C. A., (2017), Label-free detection of interleukin-6 using electrolyte gated organic field effect transistors, Biointerphases, 12(5), . https:/ / doi.org/ 10.1116/ 1.4997760

Original publication available at:

https:// doi.org/ 10.1116/1.4997760

Copyright: AIP Publishing

http:// www.aip.org/ 


\title{
Label-free detection of Interleukin-6 using Electrolyte Gated Organic Field Effect Transistors
}

Chiara Diacci, Marcello Berto, Michele Di Lauro, Elena Bianchini, Marcello Pinti, Daniel T. Simon, Fabio Biscarini and Carlo A. Bortolotti*

Chiara Diaccia), Marcello Berto ${ }^{\text {b) }}$, Michele Di Lauro, Elena Bianchini and Marcello Pinti

Università di Modena e Reggio Emilia, Dipartimento di Scienze della Vita, via Campi 103, 41125 Modena, Italy

\section{Daniel T. Simon}

Laboratory of Organic Electronics, Department of Science and Technology, ITN, Linköping University, S-601 74 Norrköping, Sweden

\section{Fabio Biscarini and Carlo A. Bortolottic)}

Università di Modena e Reggio Emilia, Dipartimento di Scienze della Vita, via Campi 103, 41125 Modena, Italy

a) Present address: Laboratory of Organic Electronics, Department of Science and Technology, ITN, Linköping University, S-601 74 Norrköping, Sweden

b) Present address: Dipartimento di Scienze Biomediche e Chirurgico Specialistiche, Università di Ferrara, Via Fossato di Mortara 17, 44121 Ferrara, Italy

c) Electronic mail: carloaugusto.bortolotti@unimore.it

\begin{abstract}
Cytokines are small proteins that play fundamental roles in inflammatory processes in the human body. In particular, Interleukin (IL)-6 is a multifunctional cytokine, whose increased levels are associated with infection, cancer and inflammation. The quantification of IL-6 is therefore of primary importance in early stages of inflammation and in chronic diseases, but standard techniques are expensive, time-consuming and usually rely on fluorescent or radioactive labels. Organic electronic devices, and in particular Organic Field-Effect Transistors (OFETs) have been proposed in the last years as novel platforms
\end{abstract}


for label-free protein detection, exploiting as sensing unit surface-immobilized antibodies or aptamers. Here we report two Electrolyte-Gated OFETs biosensors for IL-6 detection, featuring monoclonal antibodies and peptide aptamers adsorbed at the gate. Both strategies yield biosensors that can work on a wide range of IL-6 concentrations and exhibit a remarkable Limit of Detection of 1pM. Eventually, EGOFETs responses have been used to extract and compare the binding thermodynamics between the sensing moiety, immobilized at the gate electrode, and IL-6.

\section{INTRODUCTION}

Interleukin (IL)-6 is a pleiotropic cytokine with context-dependent pro- and antiinflammatory properties. ${ }^{1}$ It is a small glycoprotein produced by a broad variety of cell types, including cells of the innate and adaptive immune system as well as nonleukocytes, in response to several stimuli, particularly representing tissue damage or stress. $^{2}$ IL-6 is a multifunctional cytokine that orchestrates innate and adaptive immunity, induces the acute phase response, and is involved in bone metabolism by acting on osteoclasts and promoting osteoclastogenesis, as well as in the control of vascular permeability by inducing the production of vascular endothelial growth factor. ${ }^{2}$ It represents a critical cytokine in infection, cancer and inflammation, and it has been demonstrated that increased IL-6 levels are associated with disease settings such as several chronic inflammatory pathologies and cancer, in contrast to the relative low (1-5 $\mathrm{pg} / \mathrm{mL}$ ) physiological concentrations found in human serum. ${ }^{1}$ Moreover, increased levels

of circulating IL-6 are commonly used as indicator of inflammaging. ${ }^{3}$ Besides serving as 
a biomarker for inflammation processes, IL- 6 is now being targeted by therapeutic strategies to inhibit its pathway.

ELISA and western blotting are currently the state-of-the-art techniques for detecting IL- 6 in bodily fluids, ${ }^{4,5}$ since these techniques can provide a sensitivity in the sub pg/ml range of concentration. ${ }^{6}$ Nevertheless, both techniques are time consuming, expensive and require bulky equipment, making them difficult to transfer at the point-ofcare (POC). Electrochemical sensors based on polyelectrolyte nanoparticles loaded with ferrocene molecules can also provide good sensitivity (units of $\mathrm{pg} / \mathrm{ml}$ ), but they need sophisticated procedures. ${ }^{6,7}$ The last years have witnessed a large number of studies attempting at developing label-free biosensors alternative to currently used platforms based on optical response. ${ }^{8-15}$ As for IL-6, the latest reported biosensors that allow for a real-time monitoring of the cytokine levels without requiring secondary antibodies are immunoassays based on Graphene Oxide Field Effect Transistors, ${ }^{5}$ showing sensitivity ranging from 4.7 to $300 \mathrm{pg} / \mathrm{ml}$, Surface Plasmon Resonance ${ }^{16}$ or micro-electromechanical silicon based devices, ${ }^{17}$ with sensitivity that can be as low as about $5 \mathrm{pg} / \mathrm{mL}^{5}$ and dynamic ranges that span about two orders of magnitude. Optical methods based on labelled antibodies ${ }^{18}$ were also proposed, with a dynamic range of three orders of magnitude. Organic Field Effect Transistors are emerging as solid alternatives to both the standard commercial platforms and the innovative sensing strategies described above: ${ }^{10,19-24}$ their main advantage lies in the low-cost of fabrication and compatibility with large-area and ambient-temperature processing technologies including the highly desirable fabrication via spray, ink-jet and even roll- to-roll printing. In particular, Electrolyte Gated Organic Field-Effect Transistors (EGOFETs) are 
emerging as key players in the field of biosensing and among the most valid candidates for POC monitoring. ${ }^{11,12,15,21,25-27}$ We have recently demonstrated EGOFET-based biosensors for cytokines IL-4 ${ }^{12}$ and TNF-alpha: ${ }^{15}$ the working principle is that immunorecognition events at the gate electrode between the surface-immobilized antibody and the corresponding antigen in solution result in concentration-dependent changes in the current flowing between source and drain, due to the capacitive coupling between the organic semiconductor channel and the gate. To endow biosensors with high sensitivity and selectivity, monoclonal antibodies (Abs) are usually the binding molecule of choice to be part of the core sensing unit of the device. Although Abs still represent the golden standard for immunoassays, artificial non-antibody binders with affinity comparable to those of Abs but ensuring higher reproducibility, robustness and control over sequence are being developed. ${ }^{28-31}$ Besides DNA-based aptamers, ${ }^{10,30,32}$ protein scaffold (also referred to peptide aptamers) have been reported to successfully replace antibodies in biosensing applications ${ }^{33-37}$.

Here, we describe two EGOFET-based biosensors for detection of IL-6 with functionalized gate, one based on immobilized monoclonal Abs, and the other featuring immobilized peptide aptamers (Affimers ${ }^{\mathrm{TM}}$ ). Both devices exhibit a Limit of Detection (LOD) as low as $1 \mathrm{pM}$, which corresponds to $\sim 20 \mathrm{pg} / \mathrm{mL}$, i.e. falling in the physiological range of IL-6 levels in solution. Moreover, the devices respond across four orders of magnitude. Our configuration allows us to investigate the thermodynamics of the biorecognition events between the cytokine and its immobilized partner. We find that the particular monoclonal $\mathrm{Ab}$ and $\mathrm{Affimer}{ }^{\mathrm{TM}}$ used in the present paper exhibit comparable 
affinity for IL-6, although differences in the electrostatic contributions to the free energy of binding will be described.

\section{EXPERIMENTAL}

\section{A. Device Fabrication}

The used Test Patterns (TPs) $\left(1 \mathrm{~cm}^{2}\right.$ total area) were purchased from "Fondazione Bruno Kessler" (FBK, Trento, Italy). They feature 4 Source-Drain interdigitated electrodes with $W / L=2000$ (channel length $\mathrm{L}=15 \mu \mathrm{m}$, channel width $\mathrm{W}=30 \mathrm{~mm}$ ) patterned by photolithography and lift-off. The Au electrodes are $50 \mathrm{~nm}$ thick with a few $\mathrm{nm}$ of $\mathrm{Cr}$ adhesive layer on a quartz substrate. Before the semiconductor deposition, TPs were cleaned following the standard procedure: (i) a first rinse with acetone $(10 \mathrm{ml})$ to remove the photoresist layer, (ii) drying with nitrogen flow, (iii) washing again in hot acetone for 15 min, and (iv) drying with nitrogen. A final rinse with water was done before the semiconductor deposition. Pentacene, purchased from Sigma-Aldrich, was deposited by thermal sublimation in high vacuum on quartz TPs held at room temperature (base pressure $2 \times 10^{-8} \mathrm{mbar}$, rate $\left.7 \AA / \mathrm{min}\right)$. Pentacene film was $15 \mathrm{~nm}(\approx 10$ monolayers $)$ for all samples. ${ }^{15}$

\section{B. Gate functionalization}

Before the functionalization, gold wire $\left(0.8 \mathbf{~ m m}\right.$ diameter, $7 \mathbf{~ m m}^{2}$ active area) was cleaned following the standard procedure: 4 hours in hot $\mathrm{KOH}(2.5 \mathrm{M})$, a rinse with abundant water and then 2 hours in hot concentrated $\mathrm{H}_{2} \mathrm{SO}_{4}$. The Antibody immobilization strategy consists of a first incubation of the gold gate electrode in a Phosphate Buffer Saline (PBS $50 \mathrm{mM}, \mathrm{pH}$ 7.4) of His-tagged Protein $\mathrm{G}(5 \mathrm{mg} / \mathrm{ml})$ for half an hour at room 
temperature and then a rinse with PBS; after Protein G, the procedure is repeated with an anti-IL-6 $(0.1 \mathrm{mg} / \mathrm{ml})$ solution for 1 hour and a final rinse with PBS. ${ }^{12,15}$ In the case of Affimer-based biosensor, the gate electrode functionalization procedure is composed by two steps: incubation of the gold electrode in Affimer solution $(0.25 \mathrm{mg} / \mathrm{ml})$ overnight, a rinse with PBS and immersion in a Bovine Serum Albumin (BSA) solution $(100 \mu \mathrm{g} / \mathrm{ml})$ for $30^{\prime}$ to passivate bare gold surface spots. The last step for both is the immersion in solutions at different concentrations of IL-6 (1 pM $\div 10 \mathrm{nM})$.

\section{Electrical Characterization}

Electrical measurements were performed in a buffer solution (PBS $50 \mathrm{mM}$, pH 7.4, $50 \boldsymbol{\mu l})$ confined in a PDMS pool, as shown in Fig. 1. Source, drain, and gate electrodes were connected to an Agilent B2902A Source Meter Unit. All measurements were carried out at room temperature. The $I-V$ transfer characteristics were performed by sweeping the gate-source voltage $\left(V_{G S}\right)$ from 0.0 to $-0.8 \mathrm{~V}$ while leaving the drain-source voltage $\left(V_{D S}\right)$ constant at $-0.2 \mathrm{~V}$ (linear regime). The Limit of Detection (LOD) was calculated by the response of independently measured blank samples (PBS only, in absence of IL-6 in solution), and by calculating the corresponding mean and standard deviation ( $\sigma \mathrm{d})$ values. We then obtained the LOD as the concentration corresponding to a signal $S$ that is mean \pm

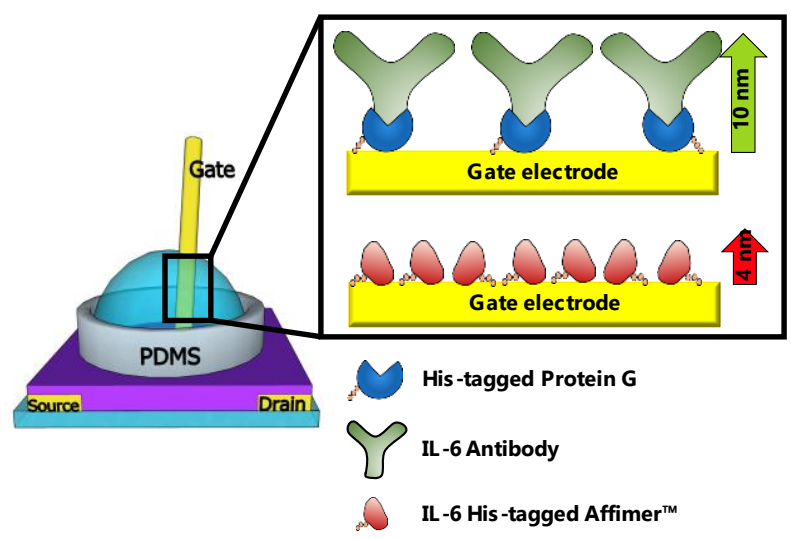


$3 \sigma \mathrm{d},{ }^{19}$ which in our case corresponds to a value, which is lower than that of the lowest investigated concentration (1 pM).

FIG. 1. Schematic experimental setup in which are highlighted Source, Drain and Gate electrodes, the PDMS pool, the organic semiconductor layer on the quartz substrate and the electrolyte (left) and the two gate functionalization strategies (right): IL-6 Antibodies are immobilized on the gate surface through His-tagged Protein G, while anti-IL-6 Affimers are directly immobilized on the gold surface by means of the His-tag.

\section{RESULTS AND DISCUSSION}

EGOFET-based immunobiosensors (i.e. featuring immobilized monoclonal $\mathrm{Ab}$ ) and peptide aptasensor (with Affimer ${ }^{\mathrm{TM}}$ adsorbed on the gate electrode) were prepared as described in the Experimental section. For both the immunobiosensor and the peptide aptasensor, the device response against different concentrations of IL-6 was monitored by recording the transfer characteristics, in the 0.0 to $-0.8 \mathrm{~V} \mathrm{~V} G$ range. The measurements were performed in PBS solutions containing increasing IL-6 concentrations, from $1 \mathrm{pM}$ to $10 \mathrm{nM}$. Fig. 2a and Fig. $2 \mathrm{~b}$ show an overlay of the transfer characteristics recorded at different [IL-6] values. From a qualitative point of view, both kinds of devices exhibit the same trend: the current $I_{D S}$ decreases upon exposure of the gate electrode to solutions containing increasing [IL-6]. Moreover, the slope of the linear part of the transfer curve also changes with [IL-6]. In order to quantitatively define the response of the biosensor, we constructed the dose curve by defining a response signal $S$, which was calculated as $S$ $=-\left(\mathrm{I}_{\mathrm{n}}-\mathrm{I}_{0}\right) / \mathrm{I}_{0}$, where $I_{n}$ is the current between source and drain $I_{D S}$ at the $n$-th 
concentration and $I_{0}$ is the current $I_{D S}$ for [IL-6] $=0 \mathrm{M}$, both extracted at the same gate voltage value. The dose curve can then be obtained by plotting $S$ versus [IL-6], as shown in Fig. 2c and Fig. 2d. Inspection of the changes in the transfer characteristics as a function of IL-6 concentration suggests that the device performances are also affected with respect to the threshold voltage $V_{t h}$ and to the transconductance $g_{m}$, the latter representing the slope of the linear region of each transfer characteristic. In particular, $g_{m}$ can be expressed as:

$$
g_{m}=\frac{W}{L} C_{G} V_{D S}
$$

where $W$ and $L$ are the channel width and length, respectively, $\mu$ is the charge carrier mobility, $C_{G}$ is the capacitance of the gate dielectric, and $V_{D S}$ is the drain voltage. ${ }^{38}$ Please note that the transconductance depends on fixed values, as the channel geometry and $V_{D S}$, and on the $\mu C_{G}$ product, which instead can be affected by binding events at the gate electrode. 
a)

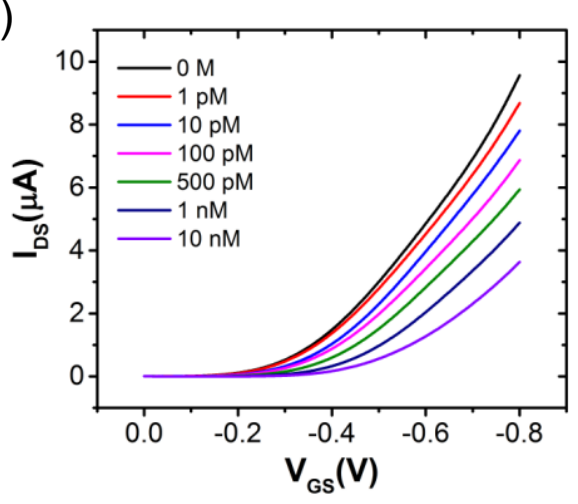

c)

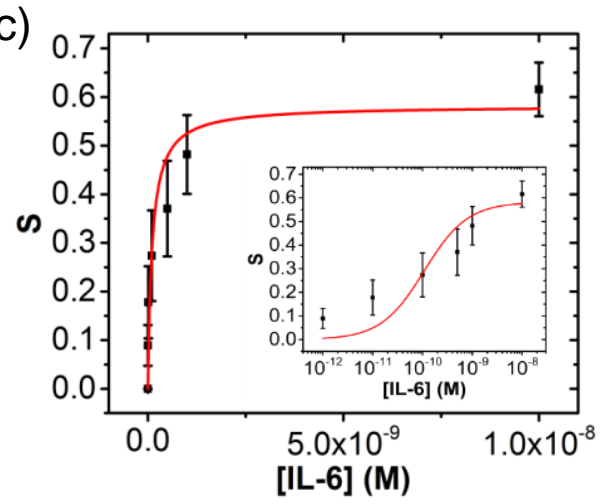

e)

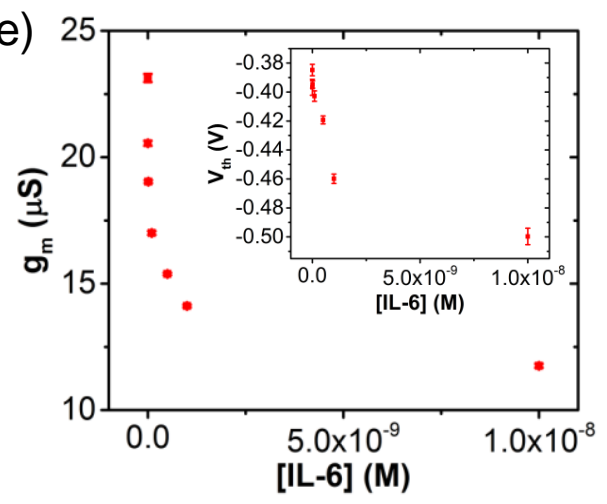

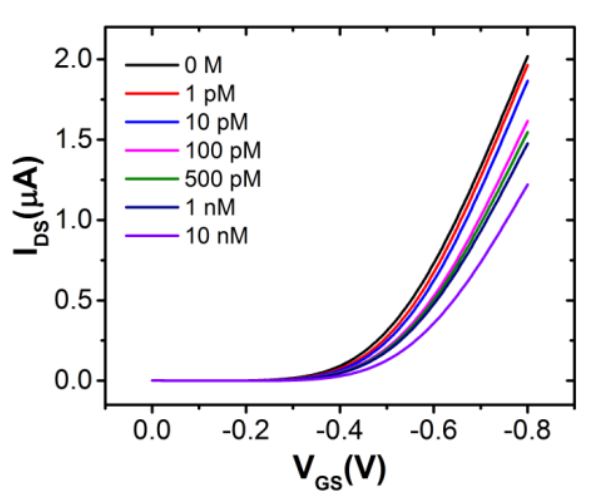

b)
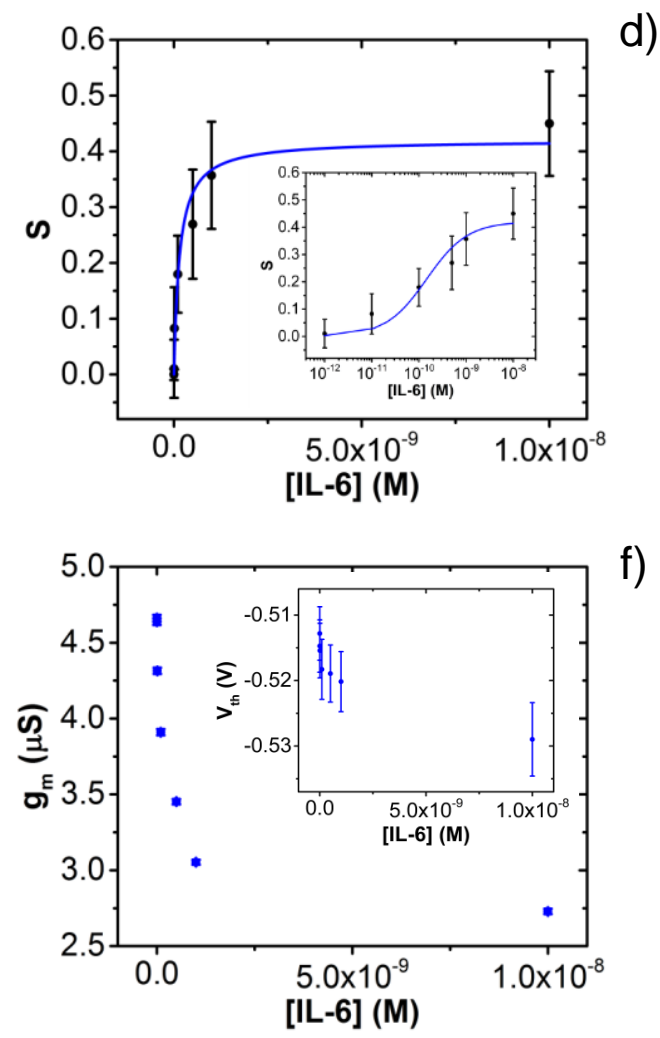

FIG. 2. Transfer characteristics of EGOFET biosensors upon exposure to different concentrations of IL-6 in PBS buffer: curves corresponding to Antibody-based biosensor (a) and Affimer-based biosensor (b). The corresponding IL-6 concentrations are reported in the legend. Dose curve $S$ vs molar concentration of IL-6, acquired at $V_{G S}=-0.8 \mathrm{~V}$, for immunosensor (c) and aptasensor (d). Inset: the dose curve with [IL-6] in logarithmic 
scale. Continuous line is the best-fit with Langmuir model. The error bars correspond to the r.m.s. of $S$ averaged over four transducing units. Transconductance $g_{m}$ as a function of IL-6 concentration for immunobiosensor in red (e) and aptasensor in blue (f). Inset: Threshold voltage $V_{t h}$ as function of [IL-6]. $g_{m}$ and $V_{t h}$ values were obtained from the linear region fit of each transfer characteristic, averaged over four transducing units and the error bars represent the r.m.s. derived from the fit.

The changes in $V_{t h}$ and $g_{m}$ for immunosensor and aptasensor as a function of [IL6] are shown in Fig. 2e and Fig. 2f, respectively. For both devices, the trends are very similar to those exhibited by the signal $S . V_{t h}$ shifts to more negative values upon IL-6 binding to the corresponding $\mathrm{Ab}$ or Affimer, and $g_{m}$ decreases accordingly. Both $V_{t h}$ and $g_{m}$ decrease rather steeply until $1 \mathrm{nM}$, and then set into a slowly decreasing linear regime. Such trends allow us to infer hypotheses on the mechanism of coupling between the binding of the antigen and the carriers in the organic semiconductive channel. The negative shift of $V_{t h}$ suggests that upon IL-6 binding there is an increase in the number of traps to charge carriers, while it is difficult to unambiguously assign the change in $g_{m}$ solely to mobility or capacitance changes. Nevertheless, panels e) and f) in Figure 2 provide evidence for the multi-parametric nature of EGOFET response, and indicate the transconductance as a solid observable, alternative to the current response, to monitor binding process. A limit of detection of $1 \mathrm{pM}$ was calculated for both Antibody- and Affimer-based biosensors. The dose curves were then fitted with a Langmuir model. Other fitting functionals, such as Hill (Supplementary eq.1) or the empirical one that we 
have previously devised and proposed ${ }^{15}$ (Supplementary eq. 2) would also yield satisfactory fits with lower reduced chi square values (see supplementary FIG.1), but that would also depend on a larger number of fitting parameters. Moreover, there might be some limitations concerning the extraction of thermodynamic parameters from such functionals: for example, in the case of the Hill model, care should be taken when trying to extract meaningful physico-chemical observables when the Hill coefficient is not unity. ${ }^{39,40}$ The applicability of the Langmuir model was also checked by rearranging the equation for the Langmuir isotherm into a linearized form and plotting [IL-6]/(S/S $\left.\mathrm{S}_{\max }\right)$ vs [IL-6] (not shown). For both plots the linear behaviour observed indicates that the Langmuir model can be used to describe the equilibrium binding of IL-6 to the gate electrodes, and therefore to extract the affinity constant $K_{a}$. We obtain the following values from the dose curves in Fig. $2: \mathrm{K}_{\mathrm{a}, \mathrm{Ab}}=9.1 \pm 7.010^{9}$ for the interaction between the surface immobilized $\mathrm{Ab}$ and IL-6, while we get $\mathrm{K}_{\mathrm{a}, \mathrm{Aff}} 6.9 \pm 3.410^{9}$ for the Affimer ${ }^{\mathrm{TM}} / \mathrm{IL}-$ 6 couple. We can therefore state that, within the assumptions underlying the use of the simple Langmuir model and its applicability to the complex bio-inorganic interfaces used here ${ }^{41}$ the surface-bound antibody and Affimer ${ }^{\mathrm{TM}}$ used here display comparable affinity for IL-6. This is in line with the same calculated LOD value and comparable sensitivity, as shown by the dose curves, for the two corresponding devices.

\section{Thermodynamics of biorecognition}

The dose curves displayed in Fig. $2 \mathrm{c}$ and Fig. $2 \mathrm{~d}$ were obtained by plotting the device response $S$ at a fixed gate voltage value $V_{G S}=-0.8 \mathrm{~V}$. We have recently demonstrated ${ }^{15}$ that the EGOFET response is modulated by the gate voltage value. It is 
apparent that the current variation upon exposure of the device to the same IL-6 solution is much higher when the device is operated at the $V_{G S}$ values in the sub-threshold regime: as $V_{G S}$ becomes more negative, the highest change in the $I_{D S}$ current observed upon IL-6 binding, $S_{\max }$, decreases significantly. Very importantly, fitting the dose curves obtained at different gate voltages, the affinity constant at the EGOFET surface $K_{a}$ turns out to be dependent on $V_{G S}$. For both Antibody- and Affimer-biosensors $K_{a}$ may decrease by up to one order of magnitude, as $V_{G S}$ is shifted from the sub-threshold regime $\left(V_{G S}=-0.3 /-0.4\right.$ V) to the accumulation regime $\left(V_{G S}=-0.7 /-0.8 \mathrm{~V}\right)$. Qualitatively, such trend is observed for both kinds of devices; to gain more insights into the molecular determinants to such changes in affinity, one can follow the approach that we recently proposed ${ }^{15}$ to compare the molar electrostatic free energy upon binding, $\Delta \mathrm{G}_{\mathrm{e}}$, for the interaction of Antibody and Affimer ${ }^{\mathrm{TM}}$ with IL-6. In particular, since the binding constant can be factorized as follows:

$$
\boldsymbol{K}_{\boldsymbol{a}}=\boldsymbol{e}^{\left(-\frac{\Delta G_{0}}{R T}\right)} \cdot \boldsymbol{e}^{\left(-\frac{\Delta G_{e}}{R T}\right)}
$$

expanding the (enthalpic part of) molar electrostatic free energy $\Delta \mathrm{G}_{\mathrm{e}}$ at the second order leads to the following equation:

$$
\Delta G_{e}=\delta Q_{m, e f f} \cdot\left(V_{G S}-V_{t h}\right)+\delta C_{m, e f f} \cdot\left(V_{G S}-V_{t h}\right)^{2}
$$

Substituting eq. 3 in eq. 2 leads to:

$$
-R T \cdot \ln K_{a}=\Delta G_{0}+\delta Q_{m, e f f} \cdot\left(V_{G S}-V_{t h}\right)+\delta C_{m, e f f} \cdot\left(V_{G S}-V_{t h}\right)^{2}
$$

Therefore, fitting the $K_{a}$ values at different $V_{G S}$ (setting $V_{t h}$ equal to its value at [IL-6] $=0$ M) with Eq. 4, one can extract the following information: $\Delta \mathrm{G}_{0}$, i.e. the standard molar free energy of Ab-antigen (or Affimer-antigen) binding at the neutral electrode interface; 
$\delta \mathrm{Q}_{\mathrm{m}}, \mathrm{eff}$, which accounts for the contribution to $\Delta \mathrm{G}_{\mathrm{e}}$ as a consequence of charge rearrangements or dipole moment changes upon binding of IL-6 to the functionalized surface; $\delta \mathrm{C}_{\mathrm{m} \text {,eff }}$, including the contribution from changes of capacitance or of polarizability following IL-6 binding to Antibody or Affimer ${ }^{\mathrm{TM}}$. Such approach was used for both biosensors to compare the thermodynamics of binding for the two different biorecognition events; the best fit parameters obtained are reported in Table I.

\begin{tabular}{|c|c|c|c|}
\hline & $\begin{array}{c}\text { Binding contribution } \\
\Delta \mathrm{G}_{0}\left(\mathrm{~kJ} \mathrm{~mol}^{-1}\right)\end{array}$ & $\begin{array}{c}\text { Charge/dipole contribution } \\
\Delta \mathrm{G}^{0}\left(\mathrm{~kJ} \mathrm{~mol}^{-1}\right)\end{array}$ & $\begin{array}{c}\text { Capacitance/polarizability } \\
\text { contribution } \\
\Delta \mathrm{G}^{0}\left(\mathrm{~kJ} \mathrm{~mol}^{-1}\right)\end{array}$ \\
\hline Antibody & $-56.2 \pm 0.1$ & $-13.5 \pm 0.4$ & $-37.4 \pm 1.7$ \\
\hline Affimer & $-57.9 \pm 0.1$ & $-10.9 \pm 1.3$ & $-16.8 \pm 3.1$ \\
\hline
\end{tabular}

TABLE I. Best fit parameters for $K_{a}$ vs $V_{G S}$ describing the thermodynamics of interaction of IL-6 with immobilized Antibody and Affimer ${ }^{\mathrm{TM}}$, respectively.

We first notice that $\Delta G_{0}$ is comparable for the two binding events. Both values are in agreement with reported standard molar free energy changes for intermolecular binding events at a surface. ${ }^{42}$

One can obtain the charge contribution to the electrostatic free energy by multiplying $\delta \mathrm{Q}_{\mathrm{m}, \text { eff }}$ by $\left(V_{G S}-V_{t h}\right)$. If we use the two extreme $V_{G S}$ values $(-0.3 \mathrm{~V}$ and $-0.8 \mathrm{~V})$, we find that for the antibody-based EGOFET, the charge contribution ranges from $-1.3 \mathrm{~kJ} \mathrm{~mol}^{-1}$ (at $V_{G S}=-0.3 \mathrm{~V}$ ) to $+8.1 \mathrm{~kJ} \mathrm{~mol}^{-1}$ (at $V_{G S}=-0.8 \mathrm{~V}$ ). This means that such contribution is negative (stabilizing) in the sub-threshold regime, but becomes higher in magnitude and positive (destabilizing the binding) in the accumulation regime. Similarly, one can 
estimate the $\delta \mathrm{C}_{\mathrm{m}, \text { eff }}$ contribution, arising from changes in capacitance and polarizability, by multiplying $\delta \mathrm{C}_{\mathrm{m}, \mathrm{eff}}$ by $\left(V_{G S}-V_{t h}\right)^{2}$. Again, using the two extreme $V_{G S}$ values yields a capacitance contribution for the antibody-based EGOFET ranging from $-0.37 \mathrm{~kJ} / \mathrm{mol}$ (at $\left.V_{G S}=-0.3 \mathrm{~V}\right)$ to $-5.9 \mathrm{~kJ} \mathrm{~mol}^{-1}\left(\right.$ at $\left.V_{G S}=-0.8 \mathrm{~V}\right)$.

As for the interaction between IL- 6 and the Affimer, the charge contribution is smaller in magnitude, but it exhibits the same behavior (slightly negative in the sub-threshold regime, positive at large and negative $V_{G S}$ values), since it ranges from $-1.0 \mathrm{~kJ} \mathrm{~mol}^{-1}$ (at $V_{G S}=-0.3 \mathrm{~V}$ ) to $+4.0 \mathrm{~kJ} \mathrm{~mol}^{-1}$ (at $V_{G S}=-0.8 \mathrm{~V}$ ). The capacitance contribution is always stabilizing: it ranges from $-0.16 \mathrm{~kJ} / \mathrm{mol}$ (at $V_{G S}=-0.3 \mathrm{~V}$ ) to $-2.56 \mathrm{~kJ} \mathrm{~mol}^{-1}$ (at $V_{G S}=-$ $0.8 \mathrm{~V})$
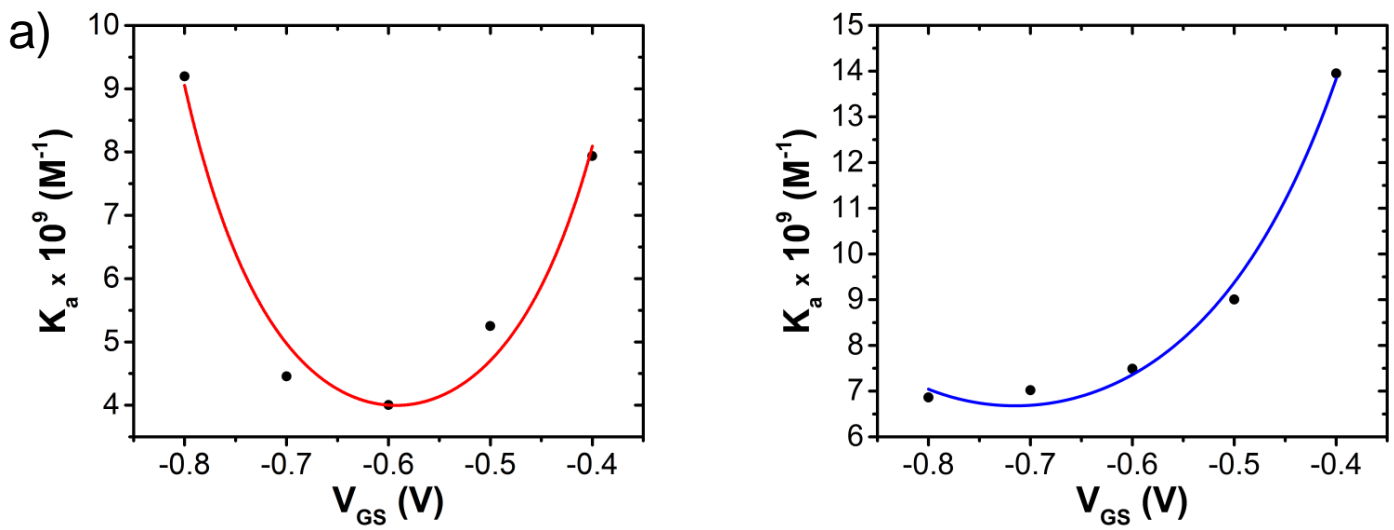

b)

FIG. 3. Dependence of affinity constant $K_{a}$ vs $V_{G S}$ for immunobiosensor (a) and peptide aptasensor (b).

The capacitance contribution $\delta \mathrm{C}_{\mathrm{m} \text {,eff }}$ is always negative (stabilizing the binding) for both couples. The charge contribution $\delta \mathrm{Q}_{\mathrm{m}, \text { eff }}$ is higher in magnitude for the interaction between IL-6 and the Antibody, and its variation in the investigated $V_{G S}$ range is more marked than that of its counterpart concerning the Affimer ${ }^{\mathrm{TM}}$. 
The sign and magnitude of the electrostatics contribution provide an explanation for the dependence of $K_{a}$ as a function of $V_{G S}$. For the Antibody, $K_{a}$ is $7.910^{9}$ at $V_{G S}=-0.4 \mathrm{~V}, 9.1$ $10^{9}$ at $V_{G S}=-0.8 \mathrm{~V}$ and its minimum value is reached at $V_{G S}=-0.6 \mathrm{~V}\left(4.010^{9}\right)$. For the Affimer ${ }^{\mathrm{TM}}, K_{a}$ is $1.310^{10}$ at $V_{G S}=-0.4 \mathrm{~V}$, and decreases to $6.910^{9}$ at $V_{G S}=-0.8 \mathrm{~V}$, as shown in Fig. 3. One more interesting consideration can be drawn when calculating the ratio between the best fit values for $\delta \mathrm{Q}_{\mathrm{m}, \mathrm{eff}}\left(-13.5 \mathrm{kC} \mathrm{mol}^{-1}\right.$ and $-10.9 \mathrm{kC} \mathrm{mol}^{-1}$ for Antibody and Affimer $^{\mathrm{TM}}$, respectively) and the Faraday constant. Such ratio is about 0.14 and 0.11 for $\mathrm{Ab}$ and Affimer, respectively, thus suggesting that binding does not lead to net charge transfer between the two biomolecules, rather only a partial charge rearrangement takes place. Therefore, we can safely infer that most of the $\delta Q_{m, e f f}$ contribution arises from the change of dipole moment at the interface.

\section{IV.SUMMARY AND CONCLUSIONS}

In this paper, we demonstrated two EGOFET-based biosensors for inflammatory cytokine IL-6, differing with respect to the biomolecule immobilized at the gate electrode to be the core sensing unit of the device. In one case, Antibodies to IL-6 were used to functionalize the gate electrode, while the second kind of device relies on the use of surface-bound Affimers $^{\mathrm{TM}}$. Both sensors exhibit LOD as low as $1 \mathrm{pM}$, which is lower than the increased levels of IL-6 in the serum of patients suffering from inflammatory response to a range of pathologies, therefore, the EGOFET biosensors described here represent a valid label-free alternative to state-of-the-art platforms for the quantification for this biomarker. 
The gate voltage $V_{G S}$ affects the device response by modulating the affinity constant $K_{a}$ between IL-6 and its surface-bound partner, by modulating the electrostatic contributions to the free energy of binding. This means that EGOFETs might be used as a tool to investigate the thermodynamics of binding between two biomolecules.

Moreover, the modulation of $V_{G S}$ can be used as an experimental handle to enhance the sensitivity of the biosensor, and bias the recognition events in the presence of interfering agents.

\section{ACKNOWLEDGMENTS}

We gratefully acknowledge IT MIUR Bilateral Project Italy/ Sweden "Poincaré" and the joint INFM-CNR Project "EOS - Organic electronics for innovative measuring instruments" for support. C.A.B. acknowledges Life Science Department through "FAR2015" and the "Fondazione di Vignola" for support.

See supplementary material at DOI: for description and comparison of different fitting models.

${ }^{1}$ C.A. Hunter and S.A. Jones, Nat. Immunol. 16, 448 (2015).

${ }^{2}$ M. Rincon, Trends Immunol. 33, 571 (2012).

${ }^{3}$ M. Pinti, V. Appay, J. Campisi, D. Frasca, T. Fülöp, D. Sauce, A. Larbi, B. Weinberger, and A. Cossarizza, Eur. J. Immunol. 46, 2286 (2016).

${ }^{4}$ S.R. Gray, M. Robinson, and M.A. Nimmo, Cell Stress Chaperones 13, 247 (2008). ${ }^{5}$ J. Huang, H. Chen, W. Niu, D.W.H. Fam, A. Palaniappan, M. Larisika, S.H. Faulkner, C. Nowak, M.A. Nimmo, B. Liedberg, and A.I.Y. Tok, RSC Adv. 5, 39245 (2015).

${ }^{6}$ H. Chen, T.K. Choo, J. Huang, Y. Wang, Y. Liu, M. Platt, A. Palaniappan, B. Liedberg, and A.I.Y. Tok, Mater. Des. 90, 852 (2016).

7 T. Li and M. Yang, Sensors Actuators, B Chem. 158, 361 (2011). 
${ }^{8}$ J.S. Daniels and N. Pourmand, Electroanalysis 19, 1239 (2007).

${ }^{9}$ K. Maehashi, T. Katsura, K. Kerman, Y. Takamura, K. Matsumoto, and E. Tamiya, Anal. Chem. 79, 782 (2007).

${ }^{10}$ M.L. Hammock, O. Knopfmacher, B.D. Naab, J.B.-H. Tok, and Z. Bao, ACS Nano 7, 3970 (2013).

${ }^{11}$ M.Y. Mulla, E. Tuccori, M. Magliulo, G. Lattanzi, G. Palazzo, K. Persaud, and L. Torsi, Nat. Commun. 6, 6010 (2015).

${ }^{12}$ S. Casalini, A.C. Dumitru, F. Leonardi, C.A. Bortolotti, E.T. Herruzo, A. Campana, R.F. de Oliveira, T. Cramer, R. Garcia, and F. Biscarini, ACS Nano 9, 5051 (2015).

${ }^{13}$ X. Luo and J.J. Davis, Chem. Soc. Rev. 42, 5944 (2013).

${ }^{14}$ R. Sharma, S.E. Deacon, D. Nowak, S.E. George, M.P. Szymonik, A.A.S. Tang, D.C. Tomlinson, A.G. Davies, M.J. McPherson, and C. Wälti, Biosens. Bioelectron. 80, 607 (2016).

${ }^{15}$ M. Berto, S. Casalini, M. Di Lauro, S.L. Marasso, M. Cocuzza, D. Perrone, M. Pinti, A. Cossarizza, C.F. Pirri, D.T. Simon, M. Berggren, F. Zerbetto, C.A. Bortolotti, and F. Biscarini, Anal. Chem. 88, 12330 (2016).

16 T.-H. Chou, C.-Y. Chuang, and C.-M. Wu, Cytokine 51, 107 (2010).

${ }^{17}$ J. Pepper, R. Noring, M. Klempner, B. Cunningham, A. Petrovich, R. Bousquet, C. Clapp, J. Brady, and B. Hugh, Sensors Actuators, B Chem. 96, 565 (2003).

${ }^{18}$ R. Kapoor and C.W. Wang, Biosens. Bioelectron. 24, 2696 (2009).

${ }^{19}$ L. Torsi, M. Magliulo, K. Manoli, and G. Palazzo, Chem. Soc. Rev. 42, 8612 (2013).

${ }^{20}$ K. Manoli, M. Magliulo, M.Y. Mulla, M. Singh, L. Sabbatini, G. Palazzo, and L. Torsi, Angew. Chemie Int. Ed. 54, 12562 (2015).

${ }^{21}$ D. Wang, V. Noël, and B. Piro, Electronics 5, 9 (2016).

${ }^{22}$ F. Torricelli, K. Manoli, E. Macchia, L. Torsi, and M. Magliulo, Org. Sensors Mater. Appl. 71 (2016).

${ }^{23}$ M.L. Hammock, A.N. Sokolov, R.M. Stoltenberg, B.D. Naab, and Z. Bao, ACS Nano 6, 3100 (2012).

${ }^{24}$ M. Magliulo, K. Manoli, E. Macchia, G. Palazzo, and L. Torsi, Adv. Mater. 27, 7528 (2015).

${ }^{25}$ S. Casalini, F. Leonardi, T. Cramer, and F. Biscarini, Org. Electron. 14, 156 (2013).

${ }^{26}$ G. Palazzo, D. De Tullio, M. Magliulo, A. Mallardi, F. Intranuovo, M.Y. Mulla, P.

Favia, I. Vikholm-Lundin, and L. Torsi, Adv. Mater. 27, 911 (2015).

${ }^{27}$ S. Lai, M. Barbaro, and A. Bonfiglio, Sensors Actuators B Chem. 233, 314 (2016).

28 A.D. Keefe, S. Pai, and A. Ellington, Nat. Rev. Drug Discov. 9, 537 (2010).

${ }^{29}$ A.B. Iliuk, L. Hu, and W.A. Tao, Anal. Chem. 83, 4440 (2011).

${ }^{30}$ M. Mascini, I. Palchetti, and S. Tombelli, Angew. Chemie Int. Ed. 51, 1316 (2012).

${ }^{31}$ S. Banta, K. Dooley, and O. Shur, Annu. Rev. Biomed. Eng. 15, 93 (2013).

${ }^{32}$ S.R. Shin, Y.S. Zhang, D. Kim, A. Manbohi, H. Avci, A. Silvestri, J. Aleman, N. Hu, T. Kilic, W. Keung, M. Righi, P. Assawes, H.A. Alhadrami, R.A. Li, M.R. Dokmeci, and A. Khademhosseini, Anal. Chem. 88, 10019 (2016).

${ }^{33}$ J.J. Davis, J. Tkac, S. Laurenson, and P. Ko Ferrigno, Anal. Chem. 79, 1089 (2007).

${ }^{34}$ J.J. Davis, J. Tkac, R. Humphreys, A.T. Buxton, T.A. Lee, and P. Ko Ferrigno, Anal. Chem. 81, 3314 (2009).

${ }^{35}$ P. Estrela, D. Paul, Q. Song, L.K.J. Stadler, L. Wang, E. Huq, J.J. Davis, P. Ko Ferrigno, and P. Migliorato, Anal. Chem. 82, 3531 (2010). 
${ }^{36}$ H. Sun and Y. Zu, Molecules 20, 11959 (2015).

${ }^{37}$ K. Škrlec, B. Štrukelj, and A. Berlec, Trends Biotechnol. 33, 408 (2015).

${ }^{38}$ K. Melzer, M. Brändlein, B. Popescu, D. Popescu, P. Lugli, and G. Scarpa, Faraday Discuss. 174, 399 (2014).

${ }^{39}$ S. Goutelle, M. Maurin, F. Rougier, X. Barbaut, L. Bourguignon, M. Ducher, and P. Maire, Fundam. Clin. Pharmacol. 22, 633 (2008).

${ }^{40}$ R. Gesztelyi, J. Zsuga, A. Kemeny-Beke, B. Varga, B. Juhasz, and A. Tosaki, Arch. Hist. Exact Sci. 66, 427 (2012).

${ }^{41}$ G. Oliviero, S. Federici, P. Colombi, and P. Bergese, J. Mol. Recognit. 24, 182 (2011).

${ }^{42}$ G. Zeder-Lutz, E. Zuber, J. Witz, and M.H. Van Regenmortel, Anal. Biochem. 246, 123 (1997). 